\title{
Documentos
}

\section{Ablación endometrial por histeroscopia: resultados, prevención y manejo de complicaciones}

\author{
Aníbal Scarella $C .^{1,2}$, Verónica Chamy $P .^{1}$, Hugo Sovino $S^{2}$ \\ 1 Departamento de Ginecología y Obstetricia, Facultad de Medicina, Universidad de Valparaíso. ${ }^{2}$ Instituto de \\ Investigación Materno Infantil (IDIMI), Facultad de Medicina, Cede Centro, Universidad de Chile.
}

\section{RESUMEN}

A mediados de la década de los 80 , se introdujeron procedimientos que eliminan el espesor completo del endometrio como tratamiento del sangrado menstrual profuso. A pesar de que en el mundo, el número y la popularidad de estas técnicas, conocida como ablación endometrial, se ha incrementado ostensiblemente, en nuestro país continúa siendo una herramienta poco difundida y subutilizada. Este artículo aborda de manera crítica la racionalidad, indicaciones y complicaciones, de la ablación endometrial en el manejo del sangrado menstrual profuso, considerando su utilidad clínica frente a otras alternativas de tratamiento.

\section{PALABRAS CLAVES: Ablación endometrial, procedimientos de primera y segunda generación, evidencia, complicaciones}

\section{SUMMARY}

In the mid 80's, procedures were introduced to eliminate the entire thickness of the endometrium as treatment of heavy menstrual bleeding. Although in the world, the number and popularity of these techniques, known as endometrial ablation has increased considerably, in our country remains a little known and underutilized tool. This article critically discusses the rationale, indications and complications of the endometrial ablation in the management of heavy menstrual bleeding, considering their clinical utility over other alternative treatments.

\section{KEY WORDS: Endometrial ablation, first and second generation procedures, evidence, complications}

\section{INTRODUCCIÓN}

El sangrado menstrual profuso o menorragia, definido como la pérdida sanguínea de $80 \mathrm{ml}$ o más por ciclo menstrual, es un problema importante de salud en la mujer (1). Estudios poblacionales señalan que entre un $10-35 \%$ de las mujeres premeno- páusicas reportan episodios de menorragia (2-4) y representa un $5-20 \%$ de las consultas de mujeres en edad reproductiva al ginecólogo general $(5,6)$. Se ha demostrado que el sangrado menstrual profuso tiene un impacto mayor en la calidad de vida y el bienestar biopsicosocial de las mujeres (1).

Se considera el manejo médico como la primera 
línea de tratamiento en mujeres premenopáusicas sin patología uterina. Dentro de ellas, el dispositivo intrauterino medicado con levonorgestrel (DIU-LNG) es la opción más efectiva si se busca un tratamiento a mediano plazo (7-10). Otras alternativas terapéuticas son los fármacos anti-fibrinolíticos (11), antiinflamatorios $(12,13)$ y el tratamiento hormonal con anticonceptivos orales $(12,14,15)$. La elección del tratamiento dependerá de diversos factores incluyendo la preferencia de la pacientes, planes reproductivos y contraindicaciones (1).

El tratamiento quirúrgico del sangrado menstrual profuso a menudo sigue al fracaso o inefectividad del tratamiento médico. Se estima que 2 de cada 3 pacientes buscará un tratamiento definitivo a dos años plazo (16). La histerectomía se considera el estándar como tratamiento quirúrgico del sangrado menstrual con una tasa de éxito primaria del $100 \%$ y altos niveles de satisfacción (16). Sin embargo, tal intervención se asocia a estadías hospitalarias prolongadas, convalecencia entre 4-6 semanas, complicaciones mayores entre un 3,6 a un $11 \%$ y una mortalidad cruda de 3,8/1000 intervenciones (17-19). Estos resultados han motivado a el desarrollo de técnicas mínimamente invasivas que disminuyan tales efectos adversos sin disminuir éxito de la intervención.

A mediados de la década de los 80 , se introdujeron técnicas que eliminan el espesor completo del endometrio; ya sea por escisión con un asa electroquirúrgica o mediante energía térmica capaz de producir necrosis del espesor completo del endometrio $(20,21)$. La popularidad de tales técnicas se ha incrementado ostensiblemente y es por ello, que en los últimos veinte años, el número de ablaciones endometriales $(\mathrm{AE})$ ha aumentado en desmedro del total de histerectomías (8), representando para el Reino Unido un $60 \%$ de las intervenciones por menorragia (Figura 1) (22).

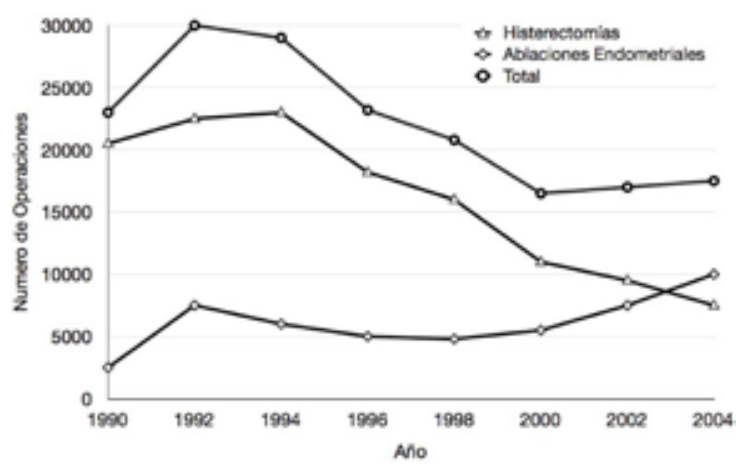

Figura 1. Tendencia en el número de histerectomías, ablaciones endometriales por sangrado uterino profuso en el Reino Unido.
El objetivo de esta revisión es aclarar las ventajas de la AE por histeroscopía frente a otras técnicas quirúrgicas, revisar las complicaciones considerando las estrategias para prevenirlas y tratarlas adecuadamente.

\section{GENERALIDADES, INDICACIONES Y FACTO- RES PRONÓSTICOS}

Se define $A E$ (8) como la destrucción quirúrgica del epitelio endometrial. Para ello, se han descrito técnicas de primera y segunda generación. Las primeras comprenden la $\mathrm{AE}$ bajo visualización directa con instrumental electroquirúrgico e incluye: resectoscopía endometrial con asa (RECA), rollerball o con láser, que han sido previamente descritas en la literatura (23). Las técnicas de segunda generación o "ciegas", dependen de un dispositivo que se inserta en la cavidad uterina y que entrega la energía para destruir uniformemente el endometrio. Las tecnologías de segunda generación aprobadas por la FDA para el uso en Estados Unidos (EEUU)

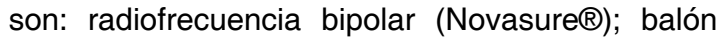
con líquido caliente (ThermaChoice $\AA$ ); crioterapia (Her Option®); dispositivo con circulación de agua caliente (Hydro ThermAblator $\AA$ ), y microonda (Microwave Endometrial Ablation).

Los resultados de las distintas técnicas de $A E$ comparadas con la histerectomía han sido evaluados por la colaboración Cochrane (24) y recientemente, en otra revisión sistemática de la literatura (RSL) (25). Los resultados muestran que la satisfacción para ambos abordajes al año de seguimiento es alta, sin embargo, un $13 \%$ de las AE y un $5 \%$ de las histerectomizadas se declara insatisfecha con el resultado al primer año con un OR de 2,46 (IC 95\% 1,5 - 3,9) en beneficio de la extracción uterina (25). A pesar de lo anterior, los resultados de calidad de vida no difirieron entre ambas técnicas (24). Más aún, la AE redujo el dolor postoperatorio, la duración de la cirugía en 23 minutos, la estadía hospitalaria en 5 días y el tiempo de incorporación al trabajo en 4 semanas (25). La mayoría de las complicaciones a corto plazo fueron más frecuentes en el grupo de histerectomía con un riesgo mayor de sepsis, transfusión de sangre, retención urinaria, anemia, fiebre, hematoma. No hubo diferencias entre los grupos para la perforación, obstrucción gastrointestinal y laparotomía como causas de complicaciones postoperatorias (24).

Dos recientes RSL que comparan técnicas de primera y segunda generación demuestran la equivalencia en cuanto a tasa de amenorrea y satisfacción de las pacientes entre las diferentes técnicas (Tabla I) $(21,25)$. El meta-análisis de la 
Tabla I

RESULTADOS DE LAS DIFERENTES TÉCNICAS DE ABLACIÓN ENDOMETRIAL (21)

\begin{tabular}{lcccc}
\hline Técnica & $\begin{array}{c}\text { Duración cirugía } \\
(\mathrm{min})\end{array}$ & $\begin{array}{c}\text { Satisfacción de } \\
\text { paciente (\%) }\end{array}$ & $\begin{array}{c}\text { Tasa amenorrea } \\
\text {-hipomenorrea } \\
(\%)\end{array}$ & $\begin{array}{c}\text { Requerimiento de cirugía } \\
\text { complementaria } \\
(\%)\end{array}$ \\
\hline Resección con asa & $10-20$ & 93,9 & 91,5 & 10,5 \\
Rollerball & $13-25$ & 97,1 & 84,2 & 24,5 \\
Ablación con láser & 30 & 89,1 & 72,5 & 16,6 \\
Ablación con balón térmico & $20-28$ & 88,6 & 81,4 & 15,3 \\
Balón de radiofrecuencia & 4 & 88,3 & 85,7 & 16,2 \\
Ablación con microondas & 2 & 98,4 & 86,9 & 8,6 \\
Hydro ThermAblator® & 10 & 97,8 & 76,6 & 10,1 \\
Crio ablación & 10 & 91,2 & 84,6 & 9,3 \\
\hline
\end{tabular}

colaboración Cochrane demostró tasas similares a los 2 años de cirugía complementaria ( $21 \%$ contra $25 \%$, OR 0,9; IC 95\% 0,6-1,4) o histerectomía (14\% versus $19 \%$, OR 0,8 ; 95\% IC 0,5-1,3) por sangrado uterino anormal (21). Aunque en ambas técnicas las complicaciones son infrecuentes, en promedio las técnicas de primera generación se asociaron a un significativo incremento del tiempo operatorio (14,5 min.), sobrecarga de fluidos $(1,43$ contra $0 \%)$, perforación uterina $(1,18$ contra $0,2 \%)$, laceraciones cervicales $(1,43$ contra $0,13 \%)$ y hematometra $(1,08$ contra $0,34 \%)$; mientras que las de segunda generación hubo mayor falla de equipo, nauseas y vómitos $(21,25)$. En las últimas, la longitud uterina $(\geq 8 \mathrm{~cm})$, la presencia de miomas o pólipos fueron fuertes predictores de insatisfacción de las pacientes. Tal relación no fue observada para las técnicas de primera generación. La comparación de las técnicas de primera generación entre sí, tampoco demostró diferencias en la tasa de amenorrea/hipomenorrea, aunque la ablación con Rollerball fue 7 minutos más corta que la RECA (21).

En base a la información señalada es que el Colegio Americano (ACOG) y el Real Colegio de Obstetras y Ginecólogos (RCOG) $(1,26,27)$ hacen las siguientes recomendaciones en cuanto a la $\mathrm{AE}$ : esta indicada en mujeres con menorragia sin respuesta al tratamiento médico y que no deseen fertilidad. Esta indicada como primera opción en mujeres con menorragia tras la discusión de los riesgos, beneficios y de otras opciones terapéuticas. Las mujeres en edad fértil deben ser prevenidas sobre la necesidad del uso de un método anticonceptivo tras la realización de la AE. La AE debe considerarse, como opción sobre la histerectomía, cuando las mujeres tengan un útero de tamaño $<10$ semanas de embarazo y/o con patología miomatosa intraca- vitaria $\leq 3 \mathrm{~cm}$ de diámetro (en este último caso con técnica de primera generación).

Las mismas asociaciones señaladas establecen como contraindicaciones absolutas de la $\mathrm{AE}$ : el embarazo, el diagnóstico o la sospecha de hiperplasia o cáncer de endometrio, el deseo de preservar la fertilidad y la infección pélvica activa. Se señala la postmenopausia y las anomalías uterinas congénitas como contraindicaciones relativas de la $A E$ en vista del mayor riesgo de perforación $(1,26)$.

\section{PREPARACIÓN ENDOMETRIAL}

Se sabe que el éxito y la facilidad técnica de una $A E$ aumenta si se realiza con un endometrio delgado o atrófico (28). Este objetivo puede lograrse en 3 maneras: programando el procedimiento en fase proliferativa inicial; realizando un legrado endometrial preoperatorio, que evita los retrasos y los costos implícitos del tratamiento médico, o mediante terapia hormonal destinada a inducir adelgazamiento endometrial, comúnmente realizada con análogos de la hormona liberadora de gonadotropina (aGnRH), danazol o progestágenos $(28,29)$.

Una RSL que compara diferentes terapias hormonales para adelgazamiento del endometrio previo a la $A E$ de primera generación, concluyó que aGnRH (30-60 días previos a la cirugía) resulta en un significativo adelgazamiento del endometrio y aumenta la probabilidad de que la cirugía alcanzará la amenorrea al compararlo con placebo (29). Resultados similares han sido demostrados con el tratamiento preoperatorio con danazol (600-800 $\mathrm{mg} /$ día durante 15 a 30 días) (29). La misma revisión destaca que existen pocos datos en base a ensayos clínicos aleatorizados (ECA) para evaluar la efectividad de progestágenos solos o combinados como agentes de adelgazamiento endometrial 
(29). Hasta la fecha, no hay otros estudios de calidad que objetiven los efectos de otros enfoques terapéuticos. Estudios que evalúan como resultado el grosor endometrial ecográfico o el grosor endometrial en histeroscopía sin $\mathrm{AE}$, demuestran que alternativas como el desogestrel (75 $\mathrm{\mu g} /$ día) con anastrazol vaginal (60 mg/día) por 10 días (30); anticonceptivos orales combinados con desogestrel (150 $\mu \mathrm{g} /$ día) y etinilestradiol (30 $\mu \mathrm{g} /$ día) por 1 ciclo (31); o la gestrinona (2,5 mg dos veces por semana) por 4 semanas (32) son métodos efectivos para adelgazar el endometrio, aunque se requieren de ECA previo a su uso en paciente candidatas a AE.

En las técnicas de segunda generación existe la instrucción de los fabricantes de los dispositivos de preparar el endometrio con aGnRH. Las excepciones son: la $A E$ con balón térmico (ThermaChoice ${ }^{\circledR}$ ) que puede ser preparado con legrado (33), o la ablación por radiofrecuencia bipolar (Novasure®) que no requieren tratamiento previo, ya que el dispositivo se ajusta a diferentes profundidades de tejido (34).

\section{PREPARACIÓN CERVICAL}

Dispositivos $\leq 5 \mathrm{~mm}$ habitualmente no requieren de dilatación cervical (35). Sin embargo, pacientes premenopáusicas que si requieren dilatación cervical, habitualmente son premedicadas con un agente osmótico (laminaria) o una prostaglandina (PG) (36). Una RSL que incluye 10 ECA, comparando misoprostol contra placebo, demostró que en mujeres premenopáusicas, el uso de PG se asoció con significativa mayor dilatación cervical inicial $(2,6 \mathrm{~mm}$; IC $95 \% 1,73$ - 3,54), una menor necesidad de dilatación cervical (42,6 contra 71,7\%; RR 0,61; IC 95\% 0,51 - 0,73$)$ y una menor tasa de laceración cervical (2 contra 11\% RR 0,22; 95\% IC 0,09 - 0,56). Tal estudio reporta un incremento de efectos secundarios menores (sangrado vaginal, cólicos abdominales y fiebre) (37). De cada cuatro mujeres que recibieron misoprostol, una mujer evitó la necesidad de una mayor dilatación cervical y 12 mujeres una laceración cervical. Tales resultados no fueron observados en posmenopáusicas en esta RSL, diferencia atribuible, según los autores, al hipoestrogenismo característico de estas pacientes (37). Oppegaard y cols (38), ratifican esta hipótesis en un estudio donde se comparan pacientes posmenopáusicas tratadas con 25 $\mu \mathrm{g}$ estradiol vaginal o placebo por 2 semanas y misoprostol $1000 \mu \mathrm{g}$ la noche previa a la histeroscopía, demostrando que el uso de estradiol preoperatorio aumenta la dilatación cervical y disminuye el dolor intraoperatorio (38).

Existe gran variabilidad en las dosis y la vía de uso del misoprostol en la literatura. La dosis ópti- ma no ha sido establecida aunque la mayoría de las publicaciones fluctúan entre 200-400 $\mu \mathrm{g}$ (37). Dosis mayores no serían necesarias (39). También existe debate en relación a la vía de administración. Choksuchat demuestra que $400 \mu \mathrm{g}$ de misoprostol oral presenta resultados similares que $200 \mu \mathrm{g}$ administrados por vía vaginal en la dilatación cervical (39). Sin embargo, a dosis equivalentes (400 $\mu \mathrm{g})$, el misoprostol administrado por vía vaginal es más eficaz que por vía oral (40). En consideración de esta información, recomendamos la administración en mujeres premenopausias de $200 \mu \mathrm{g}$ vaginal o $400 \mu \mathrm{g}$ oral la noche previa al procedimiento.

\section{COMPLICACIONES}

Como ya se ha señalado, la histeroscopía quirúrgica, en manos de un operador capacitado, resulta ser una técnica segura y eficiente en el manejo de la menorragia. El conocimiento de las complicaciones y su manejo resulta de excepcional importancia para los ginecólogos que nos enfrentamos a esta intervención, considerando que algunas son potencialmente mortales. Las complicaciones se pueden clasificar en: relacionadas con el procedimiento, relacionadas con el medio de distensión, y las del postoperatorio temprano o tardío. La Tabla II resume la frecuencia de las complicaciones más prevalentes. Abordaremos a continuación las complicaciones, a nuestro parecer más importantes, teniendo como referencia una $A E$ con técnica de primera generación.

\section{a. Relacionadas con el procedimiento}

a.1: Perforación uterina. De acuerdo a la Asociación Americana de Ginecólogos Laparoscopistas (AAGL) afecta al 1,1\% del total de procedimientos histeroscópicos (41), concentrándose principalmente en nulíparas, menopáusicas, cirugía con resectoscopía iterativa, retroversoflexión y en los primeros 100 procedimientos de un cirujano $(27,42)$. Aproximadamente la mitad de estas perforaciones ocurre durante la dilatación cervical (43). Durante la cirugía son de ayuda para la sospecha clínica: el excesivo sangrado, la pérdida repentina de la distensión uterina o la pérdida excesiva de medio. Aunque la visualización directa confirma el diagnóstico, no siempre es posible detectar esta complicaciones por lo que la aparición en el postoperatorio de dolor, distensión abdominal, sangrado vaginal profuso, hipotensión, hematuria o fiebre son signos de alarma que obligan a evaluar detenidamente a la paciente $(26,28,43)$. Al diagnóstico se debe detener inmediatamente el procedimiento. Pacientes estables, con lesión en el fondo uterino y sin activación eléctrica del asa, se pueden observar. De lo 
Tabla II

FRECUENCIA DE COMPLICACIONES SEGÚN TÉCNICA DE ABLACIÓN ENDOMETRIAL

\begin{tabular}{lccc}
\hline Tipo de complicación & $\begin{array}{c}\text { 1a generación } \\
(21)\end{array}$ & $\begin{array}{c}\text { 2a generación } \\
(21)\end{array}$ & $\begin{array}{c}\text { Hospital Naval } \\
\text { Viña del Mar }\end{array}$ \\
\hline Intoxicación hídrica & 1,6 & 0 & 1,6 \\
Hemorragia & 3,0 & 1,2 & 1,2 \\
Perforación & 1,3 & 0,3 & 0,6 \\
Fiebre & 1,1 & 1,0 & - \\
Laceraciones cervicales & 2,2 & 0,2 & 1,12 \\
Vómitos & 7,7 & 19 & - \\
\hline
\end{tabular}

contrario, se recomienda realizar una laparoscopia diagnóstica, o en su defecto una laparotomía, que permita descartar lesiones de víscera hueca o elementos sangrantes. En general, lesiones uterinas más de $1 \mathrm{~cm}$ deben ser suturadas $(26,28,36,43)$. a.2: Hemorragia. Es una complicación infrecuente y normalmente fácil de manejar. Son lugares habituales de sangrado el sitio operatorio y en caso de presentarse, la perforación uterina y laceraciones cervicales. La prevención se logra mediante una manipulación adecuada del cérvix y evitando un resección profunda con compromiso de miometrio o de miomas con compromiso miometrial $(20,44)$. El uso de vasopresina intracervical se ha demostrado útil en la reducción del sangramiento intraoperatorio además de facilitar la dilatación cervical y la prevención de absorción de medio de distensión $(20,45)$. A pesar de ser bien tolerada, su administración debe ser cuidadosa, evitando la inyección intravenosa que está asociada a hipotensión, bradicardia y mortalidad (46). El sangrado, sin perforación uterina, se controla habitualmente con electrocoagulación con rollerball. La persistencia del cuadro obliga a descartar una coagulopatía. La utilización de un balón de Foley con 15-30 ml de agua en su interior normalmente es útil en el intra o postoperatorio. La histerectomía siempre queda como un tratamiento radical de última opción $(26,28,36,43,47)$.

a.3: Lesiones térmicas. Efectos térmicos eléctricos puede causar lesiones en la cavidad uterina, intestino, vejiga y grandes vasos pélvicos (48). En especial, se ha reportado un riesgo aumentado de lesiones a intestino en la coagulación histeroscópica cornual, donde el miometrio es más delgado. Se recomiendo la movilización permanente del instrumento eléctrico activado evitando la elevación de la temperatura en la superficie uterina, minimizando el riesgo de esta complicación $(26,49)$. Se han descrito también, lesiones en vulva y vagina asociadas a dilatación excesiva del cérvix, la aplicación de corriente monopolar a $<2 \mathrm{~cm}$ de orificio cervical externo o por acoplamiento por capacitancia $(26,50)$.

b. Relacionadas con el medio líquido de distensión

La histeroscopía quirúrgica se realiza con un medio líquido de distensión (MLD) condicionado por el tipo de instrumental utilizado y por las preferencias del cirujano. En general, un medio de distensión presenta como características ideales: el permitir una visualización clara, ser no tóxicos, no conductivos, hipoalergénicos, de bajo costo, no hemolíticos, e iso-osmolar (51). El uso de instrumental monopolar requiere de un ambiente pobre en electrolitos para evitar la dispersión eléctrica a los tejidos circundantes $(26,51)$, incluyendo glicina al $1,5 \%$, sorbitol $2,7 \%$ con manitol $0,54 \%$, o manitol al 5\% (28). Por otra parte, la histeroscopía con asa bipolar (TCRis, Olympus y Versapoint, Gynecare) puede realizarse usando una solución salina o Ringer lactato eliminando el riesgo de hiponatremia dilucional, y disminuyendo, aunque no eliminando, el riesgo de sobrecarga de fluidos, edema pulmonar agudo y falla cardiaca congestiva $(26,43,51)$. Las características los MLD más utilizados en la AE se resumen en la Tabla III.

La prevención y el precoz reconocimiento de los síntomas asociados a las complicaciones por el uso de MLD pobres en electrolitos es de vital importancia $(1,26)$. El metabolismo de la glicina y el sorbitol se traduce en agua libre, cuya acumulación produce una hiponatremia hipervolémica. Normalmente, los mecanismos compensatorios se adaptan para mover el agua libre y cationes entre el espacios extra e intracelular. Sin embargo, la rápida y excesiva absorción de los medios de distensión, asociado al hecho que en mujeres los estrógenos conduce a una ineficiente acción de la bomba del sodio cerebral, causa un aumento en agua libre cerebral, hipoosmolaridad e hiponatremia, resultando en un potencial edema cerebral, presión intracraneal aumentada, y necrosis celular $(20,28,43)$. Además, la 
Tabla III

\section{CARACTERÍSTICAS DE LOS MEDIOS LÍQUIDOS DE DISTENSIÓN MÁS UTILIZADOS EN LA ABLACIÓN ENDOMETRIAL}

\begin{tabular}{llll}
\hline Categoría & Medio & Características & Complicaciones \\
\hline Electrolitos & Suero fisiológico & Agua & < Sobrecarga volumen \\
& Ringer & Electrolitos & Isotónico \\
& Manitol 5\% & Azúcar 6c. & Sobrecarga volumen \\
& & Metabolismo renal & Hiponatremia \\
Pobre en electrolitos & VM $\geq 100$ min. & \\
& Isoosmolar & Sobrecarga volumen \\
& Manitol-Sorbitol & Metabolismo hepático & Hiponatremia \\
& V/2 $\geq 33$ min. & Sobrecarga volumen \\
& Hipoosmolar & Hiponatremia \\
& Glicina & Amino-ácido & Hipoosmolaridad \\
& Metabolismo sérico a amonio & Hiperamonemia
\end{tabular}

AE: ablación endometrial; VM: vida media; $6 \mathrm{c}$ : 6 carbonado.

glicina se metaboliza a ácido glicólico y amoníaco que contribuyen y agravan los desórdenes descritos (28). Tal fenómeno se traduce en un cuadro progresivo donde aparecen: dolor de cabeza, nauseas, vómitos y agitación mental. La persistencia del cuadro y la falta de tratamiento oportuno lleva a estados excepcionales, pero críticos, como: ceguera, bradicardia e hipertensión, seguido de edema pulmonar, shock cardiovascular y muerte $(26,28,51)$.

Las complicaciones relacionadas con MLD muestran correlación con: cirugías con exposición de vasos arteriales o venosos, con pérdida $>1000$ cc de solución o en pacientes con patología asociada, aunque no con la duración de la cirugía (28). El uso de aGnRH o la administración de vasopresina intracervical han demostrado se útiles en la prevención de la absorción de $\operatorname{MLD}(20,29,45)$. En teoría, el manitol al 5\%, al ser isotónico, presenta menos riesgo de hipoosmolaridad $(28,43)$; en base a este criterio, la AAGL recomienda el uso de este MLD para la histeroscopía monopolar a pesar que los ECA no han demostrado tal diferencia $(52,53)$. Se requieren más estudios que respondan a esta interrogante.

El reconocimiento y tratamiento oportuno por personal capacitado ayuda a prevenir complicaciones severas y dificultades medico-legales (28). La
AAGL (54) y el ACOG (55) han desarrollado guías para el monitorización del uso de MLD. En base a ellas, recomendamos la aplicación de ciertas medidas sencillas y fáciles de implementar:

- el aire debe eliminarse de tuberías e histeroscopio previo al inicio del procedimiento, previniendo la embolia aérea.

- la hidratación pre e intraoperatoria, no debe ser excesiva.

Se recomienda el uso de bombas automatizadas con presión de trabajo fija entre $75-100 \mathrm{mmHg}$. De lo contrario, se puede utilizar el MLD a caída libre, siendo equivalente a la presión señalada cuando se ubica $90-120 \mathrm{~cm}$ sobre la paciente. Se debe designar en el pabellón una persona que supervise el balance del MLD, informando continuamente al cirujano del déficit del medio.

Para un correcto balance de los MLD, se debe considerar que las bolsas contienen 3-6\% extra de volumen. Además, se debe prevenir la absorción del medio por la ropa colocando un material plástico que recolecte en un recipiente libre el líquido sobrante.

Se recomienda un límite máximo de déficit de MLD de 1000 a $1500 \mathrm{ml}$ al utilizar soluciones pobre en electrolitos y $2500 \mathrm{ml}$ de soluciones con electrolitos.

Escapa al objetivo de esta revisión, el detalle 
del manejo de la paciente con una complicación relacionadas a MLD, sin embargo, es importante que todo médico histeroscopista y el restante personal de pabellón esté al tanto de las medias básicas de soporte y que existan mecanismos para la solicitud de ayuda oportuna. La Figura 1 sugiere un algoritmo básico de manejo.

\section{c. Complicaciones del posoperatorio}

Las complicaciones del posoperatorio (CP) pueden reconocerse meses o años después de la $\mathrm{AE}$. Sin considerar la ya analizada falla de la técnica, estas complicaciones son infrecuentes $(<1 \%$ de los procedimientos) y sólo analizaremos las generalidades de ellas. Existen publicaciones detalladas que las abordan en mayor profundidad $(56,57)$.

c.1: Colecciones intrauterinas. El objetivo de una $\mathrm{AE}$ es la destrucción quirúrgica total del endometrio. En ocasiones, tal proceso no es completamente satisfactorio, quedando remantes de endometrio que se manifiestan habitualmente como sangrado uterino pos AE. Algunas pacientes desarrollan sinequias $y / 0$ fibrosis que impiden la salida de la menstruación, desarrollando hematometra o un síndrome pos esterilización tubaria (SPET). Ambos cuadros se caracterizan por presentar dolor pélvico cíclico, goteo intermenstrual o amenorrea. La ecotomografía transvaginal muestra una colección líquida intracavitaria $(26,56)$. El hematometra presenta una incidencia de $1-2 \%$ y el manejo con una simple dilatación cervical resuelve el $90 \%$ de los casos $(23,58)$. El SPET se caracteriza por la formación de un hematometra cornual asociado a hidrosálpinx secundario a la menstruación retrograda que se acumula en la trompa de las pacientes salpingoligadas (SPL) (26). Se manifiesta en $<10 \%$ de las pacientes con AE y SLP y su manejo es normalmente refractario a la dilatación cervical y al tratamiento médico, siendo necesario la realización de una resección cornual o una histerectomía con salpingectomía bilateral para resolver la sintomatología (59).

c.2: Embarazo: La AE no es un método anticonceptivo. Las pacientes en riesgo de embarazo debie-

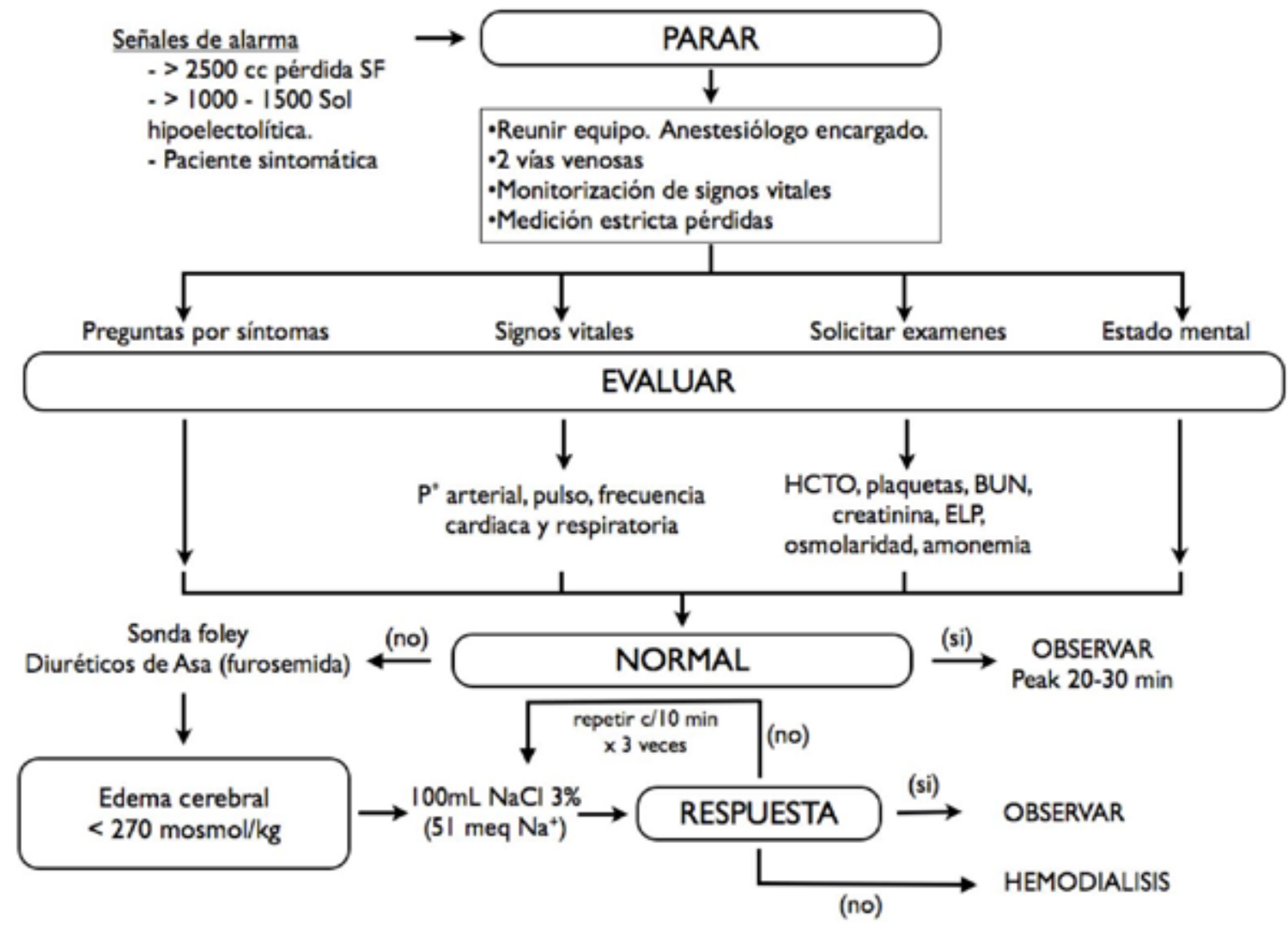

Figura 2. Esquema de manejo de absorción inadecuada de MLD. 
sen considerar una SPL o un método contraceptivo alternativo $(1,55)$. Las tasas de embarazo reportadas son bajas $(0,01-0,6 \%)(60,61)$, sin embargo, una RSL que incluye 70 casos publicados reporta un $12,9 \%$ de mortalidad perinatal, $42 \%$ de prematuridad y un $26 \%$ de placenta acreta (61), ratificando lo señalado.

c.3: Infección: Las aparición de infecciones es infrecuentes en la $\mathrm{AE}$, con una incidencia reportada de $0,3-1,6 \%(23,26)$. El ACOG no recomienda el uso de profilaxis antibiótica para este procedimiento (62). De presentarse, la mayoría de las pacientes se manifiestan con infección urinaria o endometritis, sin embargo, se han descrito casos de piometritis y abscesos tubo-ováricos. La gran mayoría de las pacientes responde al tratamiento habitual con antibióticos (56,57,63-65).

c.4: Cáncer de endometrio: Parece lógico pensar que la destrucción completa del endometrio reduce la incidencia de cáncer, sin embargo, como la remoción completa no siempre se logra, el riesgo existe. Valle y Baggish (66), en una RSL encuentran 8 casos con cáncer de endometrio tras una AE. La mayoría de ellos tenía algún factor de riesgo y todos fueron sintomáticos. Éste y otros autores en conjunto con ACOG y el RCOG recomiendan no realizar una $A E$ hasta descartar el cáncer o hiperplasia de endometrio en pacientes con anovulación crónica, endometrio engrosado o sangrado uterino anormal en $>45$ años $(1,26,66)$. La aparición de metrorragia posterior a una ablación endometrial obliga a descartar este cuadro para tratarlo adecuadamente (56).

\section{CONCLUSIÓN}

La $A E$ es una herramienta útil para el manejo de la paciente premenopáusica con menorragia. Evidencia tipo 1 respalda su uso frente a otras alternativas demostrando ser segura, relativamente fácil de implementar y con bajas tasas de complicaciones. Para un ginecólogo entrenado, presenta ventajas significativas frente a la histerectomía en cuanto al tiempo quirúrgico, estadía hospitalaria, tiempo de recuperación y complicaciones, aunque se asocia a una menor satisfacción, no presenta diferencias en cuanto a la calidad de vida al año de seguimiento. Sin embargo, este procedimiento no difiere de otros en cuanto a que es un prerrequisito conocer y manejar la técnica y sus complicaciones antes de aventurarse en la realización de tal procedimiento. Consideramos que en nuestro país la $\mathrm{AE}$ es una herramienta subutilizada, probablemente por una falta de entrenamiento, desconocimiento de sus ventajas y comprensión de sus complica- ciones. Además creemos que hay un importante campo de desarrollo para las técnicas de segunda generación cuya implementación es escasa o nula en nuestro territorio. Este artículo apunta en esa dirección y hace un llamado a impulsar el desarrollo de esta técnica quirúrgica.

\section{BIBLIOGRAFÍA}

1. National Collaborating Centre for Women's and Children's Health. Heavy menstrual bleeding. London (UK): Royal College of Obstetricians and Gynaecologists (RCOG); 2007. p. 164.

2. Cote I, Jacobs P, Cumming DC. Use of health services associated with increased menstrual loss in the United States. Am J Obstet Gynecol 2003;188:343-8.

3. Santer M, Warner P, Wyke S. A Scottish postal survey suggested that the prevailing clinical preoccupation with heavy periods does not reflect the epidemiology of reported symptoms and problems. J Clin Epidemiol 2005;58:1206-10.

4. Shapley M, Jordan K, Croft PR. An epidemiological survey of symptoms of menstrual loss in the community. Br J Gen Pract 2004;54:359-63.

5. Vessey MP, Villard-Mackintosh L, McPherson K, Coulter A, Yeates D. The epidemiology of hysterectomy: findings in a large cohort study. Br J Obstet Gynaecol 1992;99:402-7.

6. Coulter A, Bradlow J, Agass M, Martin-Bates C, Tulloch A. Outcomes of referrals to gynaecology outpatient clinics for menstrual problems: an audit of general practice records. Br J Obstet Gynaecol 1991;98:789-96.

7. Kingman CE, Kadir RA, Lee CA, Economides DL. The use of levonorgestrel-releasing intrauterine system for treatment of menorrhagia in women with inherited bleeding disorders. BJOG 2004;111:1425-8.

8. Schaedel ZE, Dolan G, Powell MC. The use of the levonorgestrel-releasing intrauterine system in the management of menorrhagia in women with hemostatic disorders. Am J Obstet Gynecol 2005;193:1361-3.

9. Kucuk T, Ertan K. Continuous oral or intramuscular medroxyprogesterone acetate versus the levonorgestrel releasing intrauterine system in the treatment of perimenopausal menorrhagia: a randomized, prospective, controlled clinical trial in female smokers. Clin Exp Obstet Gynecol 2008;35:57-60.

10. Lethaby AE, Cooke I, Rees M. Progesterone or progestogen-releasing intrauterine systems for heavy menstrual bleeding. Cochrane Database Syst Rev 2005:CD002126.

11. Lethaby A, Farquhar C, Cooke I. Antifibrinolytics for heavy menstrual bleeding. Cochrane Database Syst Rev 2000(4):CD000249.

12. Roy SN, Bhattacharya S. Benefits and risks of pharmacological agents used for the treatment of menorrhagia. Drug Saf 2004;27:75-90.

13. Lethaby A, Augood C, Duckitt K, Farquhar C. Nonsteroidal anti-inflammatory drugs for heavy menstrual bleeding. Cochrane Database Syst Rev 2007:CD000400.

14. Davis A, Godwin A, Lippman J, Olson W, Kafrissen 
M. Triphasic norgestimate-ethinyl estradiol for treating dysfunctional uterine bleeding. Obstet Gynecol 2000;96:913-20.

15. Fraser IS, McCarron G. Randomized trial of 2 hormonal and 2 prostaglandin-inhibiting agents in women with a complaint of menorrhagia. Aust $\mathrm{N} Z \mathrm{~J}$ Obstet Gynaecol 1991;31:66-70.

16. Marjoribanks J, Lethaby A, Farquhar C. Surgery versus medical therapy for heavy menstrual bleeding. Cochrane Database Syst Rev 2006:CD003855.

17. Dicker RC, Greenspan JR, Strauss LT, Cowart MR, Scally MJ, Peterson HB, et al. Complications of abdominal and vaginal hysterectomy among women of reproductive age in the United States. The Collaborative Review of Sterilization. Am J Obstet Gynecol 1982;144:841-8.

18. McPherson K, Metcalfe MA, Herbert A, Maresh M, Casbard A, Hargreaves J, et al. Severe complications of hysterectomy: the VALUE study. BJOG 2004;111:688-94.

19. Harris WJ. Complications of hysterectomy. Clin Obstet Gynecol 1997;40:928-38.

20. Istre O. Managing bleeding, fluid absorption and uterine perforation at hysteroscopy. Best Pract Res Clin Obstet Gynaecol 2009;23:619-29.

21. Lethaby A, Hickey M, Garry R, Penninx J. Endometrial resection / ablation techniques for heavy menstrual bleeding. Cochrane Database Syst Rev 2009:CD001501.

22. Reid PC. Endometrial ablation in England--coming of age? An examination of hospital episode statistics 1989/1990 to 2004/2005. Eur J Obstet Gynecol Reprod Biol 2007;135:191-4.

23. van Herendael BJ. Endometrial resection. In: Donnez J, editor. Atlas of OPERATIVE LAPAROSCOPY AND HYSTEROSCOPY. Third Edition ed. London, UK.: Informa Healthcare; 2007. p. 527-32.

24. Lethaby A, Shepperd S, Cooke I, Farquhar C. Endometrial resection and ablation versus hysterectomy for heavy menstrual bleeding. Cochrane Database Syst Rev 2000:CD000329.

25. Middleton LJ, Champaneria R, Daniels JP, Bhattacharya S, Cooper KG, Hilken NH, et al. Hysterectomy, endometrial destruction, and levonorgestrel releasing intrauterine system (Mirena) for heavy menstrual bleeding: systematic review and meta-analysis of data from individual patients. BMJ;341:c3929.

26. ACOG Practice Bulletin No. 81: Endometrial Ablation. Obstetrics \& Gynecology 2007;109(5):1233-48 0.097/01.AOG.0000263898.22544.cd.

27. ACOG practice bulletin: management of anovulatory bleeding. Int J Gynaecol Obstet 2007;72:263-71.

28. Bradley LD. Complications in hysteroscopy: prevention, treatment and legal risk. Curr Opin Obstet Gynecol 2002;14:409-15.

29. Sowter MC, Lethaby A, Singla AA. Pre-operative endometrial thinning agents before endometrial destruction for heavy menstrual bleeding. Cochrane Database Syst Rev 2002:CD001124.

30. Cicinelli E, Pinto V, Tinelli R, Saliani N, De Leo V, Cianci A. Rapid endometrial preparation for hysteroscopic surgery with oral desogestrel plus vaginal ra- loxifene: a prospective, randomized pilot study. Fertil Steril 2007;88:698-701.

31. Grow DR, Iromloo K. Oral contraceptives maintain a very thin endometrium before operative hysteroscopy. Fertil Steril 2006;85:204-7.

32. Triolo O, De Vivo A, Benedetto V, Falcone S, Antico F. Gestrinone versus danazol as preoperative treatment for hysteroscopic surgery: a prospective, randomized evaluation. Fertil Steril 2006;85:1027-31.

33. Sharp HT. Assessment of new technology in the treatment of idiopathic menorrhagia and uterine leiomyomata. Obstet Gynecol 2006;108:990-1003.

34. NovaSure. Disponible en: www.novasure.com/novasure-procedure/novasure-instructions.cfm Acceso: 5 Septiembre de 2010.

35. Readman E, Maher PJ. Pain relief and outpatient hysteroscopy: a literature review. J Am Assoc Gynecol Laparosc 2004;11:315-9.

36. Levy B, Falcone T, Falk S. Uterine perforation during gynecologic procedures. In: Basow DS, editor. UpToDate. Waltham; MA: UpToDate; 2010.

37. Crane JM, Healey S. Use of misoprostol before hysteroscopy: a systematic review. J Obstet Gynaecol Can 2006;28:373-9.

38. Oppegaard KS, Lieng M, Berg A, Istre O, Qvigstad E, Nesheim BI. A combination of misoprostol and estradiol for preoperative cervical ripening in postmenopausal women: a randomised controlled trial. BJOG 2010;117:53-61.

39. Choksuchat C. Clinical use of misoprostol in nonpregnant women: review article. J Minim Invasive Gynecol 2010;17:449-55.

40. Batukan C, Ozgun MT, Ozcelik B, Aygen E, Sahin Y, Turkyilmaz $\mathrm{C}$. Cervical ripening before operative hysteroscopy in premenopausal women: a randomized, double-blind, placebo-controlled comparison of vaginal and oral misoprostol. Fertil Steril 2008;89:966-73.

41. Hulka JF, Peterson HB, Phillips JM, Surrey MW. Operative hysteroscopy. American Association of Gynecologic Laparoscopists 1991 membership survey. J Reprod Med 1993;38:572-3.

42. Sowter M, Lethaby A, Farquhar C. A national survey of the complications of endometrial destruction for menstrual disorders: the MISTLETOE study. $\mathrm{Br} \mathrm{J}$ Obstet Gynaecol 1998;105:1341-2.

43. Paschopoulos M, Polyzos NP, Lavasidis LG, Vrekoussis T, Dalkalitsis N, Paraskevaidis E. Safety issues of hysteroscopic surgery. Ann N Y Acad Sci 2006;1092:229-34.

44. Wamsteker K, Emanuel MH, de Kruif JH. Transcervical hysteroscopic resection of submucous fibroids for abnormal uterine bleeding: results regarding the degree of intramural extension. Obstet Gynecol 1993;82(5):736-40.

45. Phillips DR, Nathanson HG, Milim SJ, Haselkorn JS, Khapra A, Ross PL. The effect of dilute vasopressin solution on blood loss during operative hysteroscopy: a randomized controlled trial. Obstet Gynecol 1996;88:761-6.

46. Hobo R, Netsu S, Koyasu Y, Tsutsumi O. Bradycardia and cardiac arrest caused by intramyometrial injection of vasopressin during a laparoscopically assisted myo- 
mectomy. Obstet Gynecol 2009;113(2 Pt 2):484-6.

47. Serden SP, Brooks PG. Treatment of abnormal uterine bleeding with the gynecologic resectoscope. J Reprod Med 1991;36:697-9.

48. Vilos GA, Brown S, Graham G, McCulloch S, Borg $P$. Genital tract electrical burns during hysteroscopic endometrial ablation: report of 13 cases in the United States and Canada. J Am Assoc Gynecol Laparosc 2000;7:141-7.

49. Duffy S, Reid PC, Sharp F. In-vivo studies of uterine electrosurgery. Br J Obstet Gynaecol 1992;99:579-82.

50. Munro MG. Mechanisms of thermal injury to the lower genital tract with radiofrequency resectoscopic surgery. J Minim Invasive Gynecol 2006;13:36-42.

51. Bradley LD, Falcone T, Falk S. Hysteroscopy: Managing fluid and gas distending media. In: Basow DS, editor. UpToDate. Waltham; MA: UpToDate; 2010.

52. Phillips DR, Milim SJ, Nathanson HG, Phillips RE, Haselkorn JS. Preventing hyponatremic encephalopathy: comparison of serum sodium and osmolality during operative hysteroscopy with $5.0 \%$ mannitol and $1.5 \%$ glycine distention media. J Am Assoc Gynecol Laparosc 1997;4:567-76.

53. Inman RD, Hussain Z, Elves AW, Hallworth MJ, Jones PW, Coppinger SW. A comparison of $1.5 \%$ glycine and $2.7 \%$ sorbitol- $0.5 \%$ mannitol irrigants during transurethral prostate resection. J Urol 2001;166:2216-20.

54. Loffer FD, Bradley LD, Brill AI, Brooks PG, Cooper JM. Hysteroscopic fluid monitoring guidelines. The ad hoc committee on hysteroscopic training guidelines of the American Association of Gynecologic Laparoscopists. J Am Assoc Gynecol Laparosc 2000;7:167-8.

55. ACOG technology assessment in obstetrics and gynecology, number 4, August 2005: hysteroscopy. Obstet Gynecol 2005;106:439-42.

56. McCausland AM, McCausland VM. Long-term complications of endometrial ablation: cause, diagnosis, treatment, and prevention. J Minim Invasive Gynecol 2007;14:399-406.

57. Cooper JM, Brady RM. Late complications of operative hysteroscopy. Obstet Gynecol Clin North Am 2000;27:367-74.

58. Hill DJ. Hematometra-A Complication of Endometrial Ablation/Resection. J Am Assoc Gynecol Laparosc 1994;1(4, Part 2):S14.

59. McCausland AM, McCausland VM. Frequency of symptomatic cornual hematometra and postablation tubal sterilization syndrome after total rollerball endometrial ablation: a 10-year follow-up. Am J Obstet Gynecol 2002;186:1274-80; discussion 80-3.

60. Vilos GA, Vilos EC, King JH. Experience with 800 hysteroscopic endometrial ablations. J Am Assoc Gynecol Laparosc 1996;4:33-8.

61. Hare AA, Olah KS. Pregnancy following endometrial ablation: a review article. J Obstet Gynaecol 2005;25:108-14.

62. ACOG practice bulletin No. 104: antibiotic prophylaxis for gynecologic procedures. Obstet Gynecol 2009;113:1180-9.

63. Amin-Hanjani S, Good JM. Pyometra after endometrial resection and ablation. Obstet Gynecol 1995;85(5 Pt 2):893-4.

64. Schlumbrecht M, Balgobin S, Word L. Pyometra after thermal endometrial ablation. Obstet Gynecol 2007; 110(2 Pt 2):538-40.

65. Garry R, Shelley-Jones D, Mooney P, Phillips G. Six hundred endometrial laser ablations. Obstet Gynecol 1995;85:24-9.

66. Valle RF, Baggish MS. Endometrial carcinoma after endometrial ablation: high-risk factors predicting its occurrence. Am J Obstet Gynecol 1998;179(3 Pt 1):569-72. 